\title{
Focal lesion at the midline of the prostate on transrectal ultrasonography: take it or leave it?
}

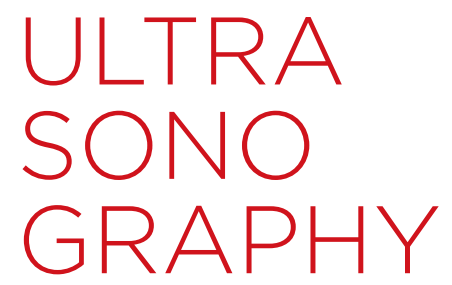

\author{
Junwoo Kim', Sung II Hwang ${ }^{1}$, Hak Jong Lee', Sung Kyu Hong², Seok-Soo Byun², \\ Sangchul Lee ${ }^{2}$, Gheeyoung $\mathrm{Choe}^{3}$ \\ Departments of ${ }^{1}$ Radiology, ${ }^{2}$ Urology, and ${ }^{3}$ Pathology, Seoul National University Bundang \\ Hospital, Seongnam, Korea
}

Purpose: The purpose of this study was to analyze the detection rate of prostate cancers from targeted biopsy specimens of midline focal lesions and to investigate the ultrasonographic findings to reduce unnecessary additional targeted biopsies.

Methods: Ninety-eight men with midline focal lesions detected on transrectal ultrasonography were enrolled. Additional targeted biopsies for midline focal lesions were performed after 12core random systematic biopsies. Correlations between the ultrasonographic characteristics of midline focal lesions and the pathologic results were analyzed.

Results: Twenty of 98 targeted biopsy cores (20.4\%) were positive for malignancy. In a univariate analysis, midline focal lesions without bulging contours $(P=0.023)$, with involved margins ( $P=0.001)$, without hypoechoic perilesional rims $(P=0.005)$, and with longer diameters $(P=0.005)$ were statistically significant for cancer detection. In a multivariate analysis, involved margin $(P=0.027)$, having longer diameter $(P=0.011)$ or absence of hypoechoic perilesional rim $(\mathrm{P}=0.025)$ made a statistically significant contribution to cancer detection.

Conclusion: Biopsy of midline focal lesions was not always non-significant in the detection of prostate cancer. Additional targeted biopsies should be considered in cases of midline focal lesions with involved margins but without hypoechoic perilesional rims.

Keywords: Prostatic neoplasms; Ultrasonography; Image-guided biopsy

\section{ORIGINAL ARTICLE}

https://doi.org/10.14366/usg. 16014 pISSN: 2288-5919 - elSSN: 2288-5943 Ultrasonography 2017;36:10-16

Received: March 9, 2016

Revised: May 1, 2016

Accepted: May 16, 2016

Correspondence to:

Sung II Hwang, MD, Department of Radiology, Seoul National University Bundang Hospital, 82 Gumi-ro 173 beon-gil, Bundang-gu, Seongnam 13620, Korea

Tel. +82-31-787-7621

Fax. +82-31-787-4011

E-mail: hwangsi49@gmail.com

This is an Open Access article distributed under the terms of the Creative Commons Attribution NonCommercial License (http://creativecommons.org/ licenses/by-nc/3.0/) which permits unrestricted noncommercial use, distribution, and reproduction in any medium, provided the original work is properly cited.

Copyright @ 2017 Korean Society of Ultrasound in Medicine (KSUM)

\section{Introduction}

Midline focal lesions of the prostate are defined as lesions located in the midline and bilaterally involving the right and left paramedian peripheral zones. Space-occupying lesions in the midline of the prostate can confound prostate cancer detection, as they can be categorized as normal anatomical structures, such as central-zone or intraprostatic median cysts, and even as noncancerous conditions, such as benign prostatic hyperplastic nodules or granulomatous prostatitis [1-3]. Therefore, midline focal lesions are known as pitfalls for prostate cancer detection.

Most guidelines on prostate cancer recommend that the initial biopsy focus mainly on the peripheral zone, especially the posterior and lateral regions [4]. Eight to 12 random systematic core

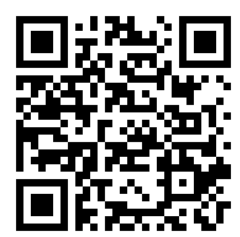

How to cite this article:

Kim J, Hwang SI, Lee HJ, Hong SK, Byun SS, Lee $S$, et al. Focal lesion at the midline of the prostate on transrectal ultrasonography: take it or leave it?. Ultrasonography. 2017 Jan;36(1)10-16. 
biopsies, focused mainly on the peripheral zone, have recently been recommended [5]. Additional midline peripheral zone biopsies in the initial biopsy protocol are not supported by most studies. However, studies have shown that $2 \%$ of patients are diagnosed with prostate cancer only after a midline biopsy is positive $[4,6]$. According to these reports, additional targeted biopsies for midline focal lesions should be generally avoided. But in some exceptional cases, additional targeted biopsies could improve cancer detection. Considering the low probability of additional cancer detection in midline biopsies, multiple factors should be evaluated to avoid unnecessary biopsy. Transrectal ultrasonography (TRUS) characterization of focal lesions could be helpful, whether performing additional biopsy or not.

Classically, prostate cancers have been described as hypoechoic lesions on ultrasonography (US) [7], related to microscopic changes of the glandular structure, increased cellularity, and alterations of the microvascular structure [8]. However, the advent of prostate-specific antigen (PSA) and high-frequency ultrasound transducers has led to an era of early prostate cancer detection, and prostate cancers have been recognized to have variable echotextures. Spajic et al. [9] reported that $60.6 \%$ of prostate cancers involved hypoechoic lesions and $31.8 \%$ involved isoechoic lesions. The sole basis of TRUS echogenicity fell in importance for determining prostate cancer. Heijmink et al. [10] reported that identification of hypoechoic nodules as prostate cancers had decreased from $17 \%-57 \%$ to $9 \%$ after the implementation of PSA testing. That study also reported that targeted biopsies for hypoechoic lesions on TRUS showed high sensitivity, but low specificity. Further characterization of focal prostate lesions was deemed necessary, beyond echogenicity. Evaluating the specific characteristics of focal lesions on grayscale US and color Doppler US (CDUS) improved prostate cancer detection in several studies [11-13]. Some studies also showed that prostate cancer patients with suspicious findings on TRUS were observed to have high probability of malignancy compared to patients without suspicious findings [14].

Therefore, identifying the US characteristics of midline focal lesions could determine whether additional targeted biopsies should be performed. In this study, we analyzed the detection rate of prostate cancers from targeted biopsy specimens of midline focal lesions, and investigated the US findings in order to reduce unnecessary additional targeted biopsies.

\section{Materials and Methods}

This study was approved by the Institutional Review Board of Seoul National University Bundang Hospital, which waived the requirement for informed consent for the use of patient data because of the retrospective design.

\section{Study Population}

From January 2005 to February 2015, 7,485 consecutive patients were referred for prostate biopsies. The indications for biopsy were abnormal digital rectal exam and/or increased PSA (greater than $3.0 \mathrm{ng} / \mathrm{mL}$ ) and/or abnormalities on previous TRUS. Retrospective review of electronic medical records and the picture archiving and communication system identified 1,124 men who underwent additional targeted biopsy for focal lesions after 12-core random systematic biopsies. Ninety-eight of these men (mean age, 67.0 \pm 7.6 years) with midline focal lesions were finally enrolled. The mean PSA level was $11.2 \pm 20.6 \mathrm{ng} / \mathrm{dL}$ (range, 0.4 to $186.8 \mathrm{ng} / \mathrm{dL}$ ).

\section{US-Guided Biopsy Procedures}

All of the examinations were performed using an iU22 ultrasound scanner (Philips, Bothell, WA, USA) equipped with a 9-4 MHz broadband curved array endocavitary transducer. US-guided biopsies were performed by using an automated gun with a core length of $2 \mathrm{~cm}$ (AceCut, TSK, Japan) after a nerve block with $10 \mathrm{~mL}$ lidocaine. Twelve extended systematic biopsies were obtained at the apex, mid-gland, and base of the prostate in the parasagittal plane ( 6 paramedian cores were obtained) and the lateral directed plane bilaterally (6 lateral cores were obtained). This was followed with additional single-core targeted biopsies for midline focal lesions. All US examinations and US-guided biopsies were performed by one of two board-certified uroradiologists (S.I.H. with 13 years of experience, H.J.L. with 17 years of experience).

\section{Analysis of US Findings}

Sonograms were reviewed in consensus by two radiologists (S.I.H. and J.K.) who were blinded to the clinical information and histologic results. The characteristics included shape (band-like, nodular), outward contour (bulging, not bulging), margins (clear, involved), presence or absence of a hypoechoic perilesional rim, presence of blood flow on CDUS, and measurement of the longest diameter (Fig. 1). The term "band-like" for lesion shape was used in cases of lesions that were neither oval nor round. "Bulging contour" was defined as a focal lesion with a mass effect on adjacent tissue. "Clear" margins indicated that the focal lesion was well delineated against the background prostate tissue. "Hypoechoic perilesional rim" was defined as the presence of a halo surrounding the focal lesion.

\section{Analysis of Biopsy Results}

Biopsy results were used as the gold standard. Each biopsy core was reviewed by a single pathologist. The patients with more than 


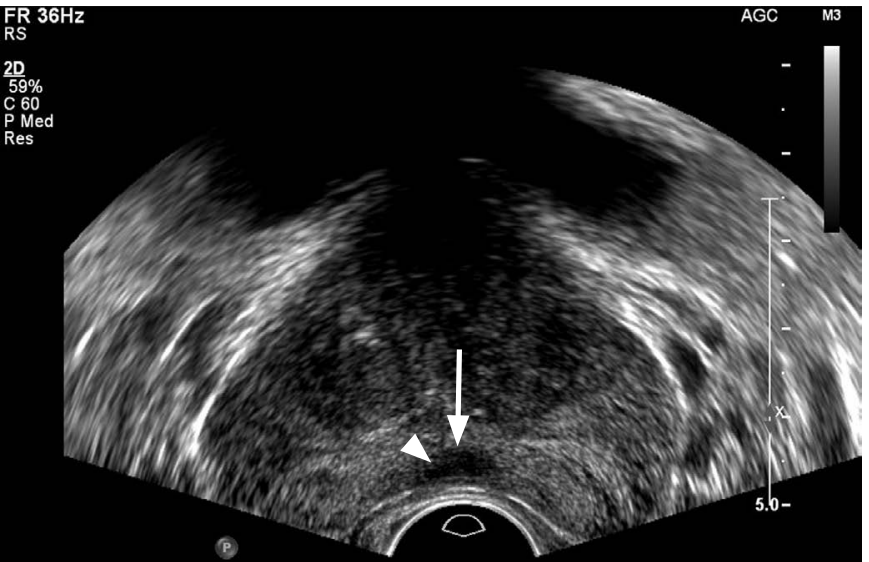

A

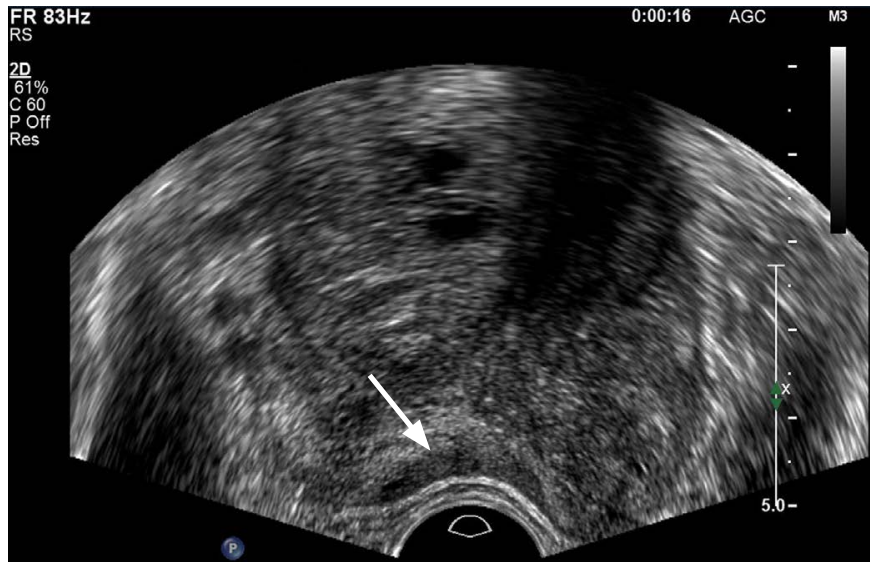

B

Fig. 1. Two examples of transrectal ultrasonography (TRUS) for prostate lesions.

A. On TRUS of a 62-year-old man, a $0.8-\mathrm{cm}$ midline focal lesion at mid-gland level is classified as a nodular lesion with a bulging contour and involved margins (arrowhead). A hypoechoic rim (arrow) is present. Targeted biopsy proved the tissue to be positive for malignancy. B. In a 70-year-old man, TRUS shows a 0.9-cm midline focal lesion (arrow) at mid-gland level. The lesion is classified as a band-like lesion without a bulging contour. The margins are clear but a hypoechoic rim is absent. A targeted biopsy proved negative for malignancy.

two cores with a Gleason score (GS) of $\geq 6$ or single core of $\geq 7$ were regarded as the clinically significant group.

\section{Statistical Analysis}

The relationship between histologic results and US findings was evaluated. Fisher exact test and a paired t test were used for univariate analysis, and multiple logistic regression was used for multivariate analysis. P-values of $<0.05$ were considered to indicate statistical significance. Statistical analysis was done with SPSS ver. 21.0 (IBM Corp., Armonk, NY, USA).

\section{Results}

\section{Biopsy Results}

Prostate cancer was detected in 45 out of 98 patients (54.1\%) by systematic and targeted biopsies. The distribution was a GS of 6 in 21 patients (46.7\%), a GS of 7 in 18 patients (40\%), and a GS of 8 or higher in six patients (13.3\%). Two patients with a GS of 6 and 24 patients with a GS of $\geq 7$ were regarded as the clinically significant group. Twenty out of 98 (20.4\%) targeted biopsy specimens were positive. In two patients, cancer was detected only in the targeted core. The results from the targeted core moved two patients into the clinically significant cancer group. There was no major biopsy related complication that prolonged the hospital stay.

\section{Univariate Logistic Regression Analysis}

Midline focal lesions without bulging contours $(P=0.023)$, with involved margins $(P=0.001)$, and with the absence of hypoechoic perilesional rims ( $P=0.005)$ were statistically significant for cancer detection by Fisher exact test. A longer diameter also resulted in increased cancer detection on univariate logistic regression $(P=0.005)$. The mean diameter of focal lesions in the cancernegative group $(7.8 \pm 2.2 \mathrm{~mm})$ were significantly shorter than in the cancer-positive group $(10.0 \pm 4.4 \mathrm{~mm})$ on the paired t test $(\mathrm{P}=0.002)$ (Table 1).

\section{Multivariate Logistic Regression Analysis}

In the multivariate logistic regression analysis, three criteria, including involved margins ( $\mathrm{P}=0.027$; odds ratio [OR], 3.770; 95\% confidence interval $[\mathrm{Cl}], 1.159$ to 12.260$)$, absence of a hypoechoic perilesional rim ( $P=0.025 ; O R, 5.039 ; 95 \% \mathrm{Cl}, 1.221$ to 20.793), and a longer diameter $(\mathrm{P}=0.011 ; \mathrm{OR}, 1.297 ; 95 \% \mathrm{Cl}, 1.062$ to 1.584$)$ were statistically significant for cancer detection (Table 2). The area under the receiver operating characteristics curve for the logistic regression model was $0.822(95 \% \mathrm{Cl}, 0.728$ to 0.917$)$ (Fig. 2). At the best diagnostic point, the logistic regression model showed an accuracy of $82.7 \%$ (sensitivity, $70.0 \%$; specificity, $85.9 \%$; cutoff value $\mathrm{P}=0.300$ ).

\section{Discussion}

Twenty out of 98 prostate cancers (20.4\%) were detected via targeted biopsy. Prostate cancer was detected solely in targeted biopsy specimens in two patients. Targeted biopsy led to moving another two clinically insignificant patients into the significant cancer group. Each of these results was similar to previous studies, 
Table 1. Evaluation of the US characteristics of midline focal lesions

\begin{tabular}{|c|c|c|c|c|}
\hline Characteristic & Benign $(n=78)$ & Malignant $(n=20)$ & Total $(n=98)$ & P-value \\
\hline Shape & & & & 0.107 \\
\hline Band-like & 11 & 6 & 17 & \\
\hline Nodular & 67 & 14 & 81 & \\
\hline Bulging contour & & & & 0.023 \\
\hline Positive & 47 & 6 & 53 & \\
\hline Negative & 31 & 14 & 45 & \\
\hline Margin & & & & 0.001 \\
\hline Clear & 65 & 9 & 74 & \\
\hline Involved & 13 & 11 & 24 & \\
\hline Hypoechoic rim & & & & 0.005 \\
\hline Positive & 40 & 3 & 43 & \\
\hline Negative & 38 & 17 & 55 & \\
\hline Color Doppler US & & & & 0.311 \\
\hline Positive & 43 & 14 & 57 & \\
\hline Negative & 35 & 6 & 41 & \\
\hline Lesion diameter & $7.8 \pm 2.2$ & $10.0 \pm 4.4$ & & 0.002 \\
\hline
\end{tabular}

${ }^{a}$ The P-values were calculated with Fisher exact test; the lesion diameter was calculated with univariate logistic regression.

Table 2. Cancer detection related to US characteristics by multivariate logistic regression

\begin{tabular}{lccc}
\multicolumn{1}{c}{ Variable } & P-value & OR & 95\% Cl \\
\hline Involved margin & 0.027 & 3.770 & $1.159-12.260$ \\
Absence of hypoechoic rim & 0.025 & 5.039 & $1.221-20.793$ \\
Lesion diameter & 0.011 & 1.297 & $1.062-1.584$ \\
Nodular shape $^{\text {a) }}$ & 0.226 & - & - \\
Presence of bulging contour $^{\text {a) }}$ & 0.544 & - & - \\
Presence of blood flow in color Doppler US $^{\mathrm{a})}$ & 0.438 & - & - \\
\hline
\end{tabular}

US, ultrasonography; OR, odds ratio; $\mathrm{Cl}$, confidence interval.

${ }^{\text {a) }}$ Variables not significant in multivariate analysis (those excluded in the equation).

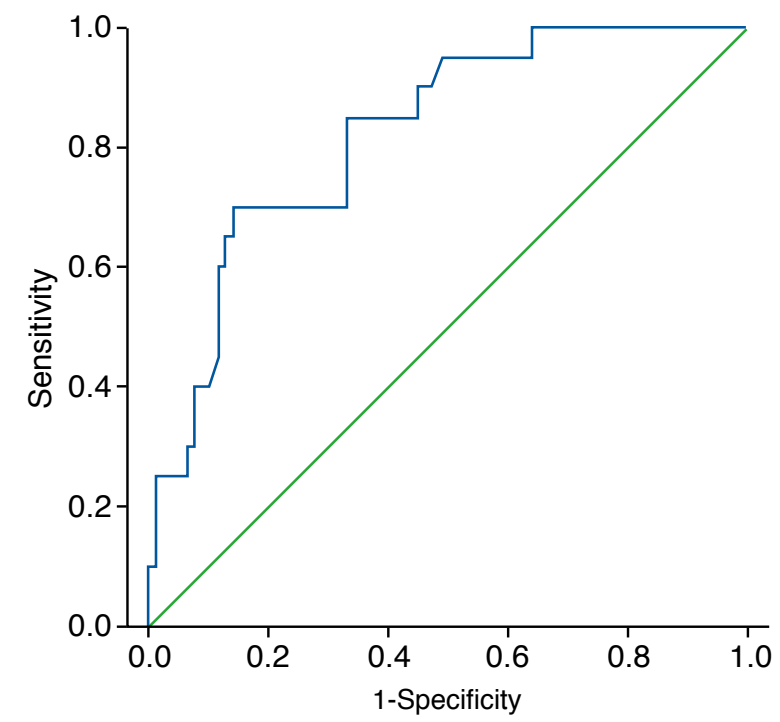

Fig. 2. Receiver operating characteristics (ROC) curve for the logistic regression model based on combination of three parameters (involved margin, absence of hypoechoic rim, and lesion diameter). The area under the ROC curve of the logistic regression model was 0.822 (95\% confidence interval, 0.728 to 0.917). 
which reported that $2 \%$ of patients were diagnosed with cancer solely due to midline biopsies $[4,6]$. If we had not performed targeted biopsies in this population, we would have missed two cases of prostate cancer or would have underestimated their clinical significance.

Based on the imaging findings in our study, midline focal lesions with involved margins ( $P=0.001)$, but without hypoechoic perilesional rims ( $P=0.005)$ and without bulging contours $(P=0.023)$, were statistically significant for cancer detection in the univariate analysis. In the multivariate analysis, midline focal lesions with involved margins $(P=0.027)$ and longer diameters $(P=0.011)$, but without hypoechoic rims ( $\mathrm{P}=0.025)$, were also statistically significant for cancer detection. To summarize these findings, midline focal lesions with infiltrative characteristics, rather than those with wellcircumscribed margination, could be proved to be prostate cancers rather than benign conditions, such as hyperplastic nodules or inflammatory lesions (Fig. 3).

The results and imaging characteristics in the present study support previous studies in two ways. First, our study re-emphasized TRUS identification of focal lesions. Although random systematic biopsy is considered the standard for the initial biopsy, some studies have reported that additional targeted biopsies for TRUS-identified

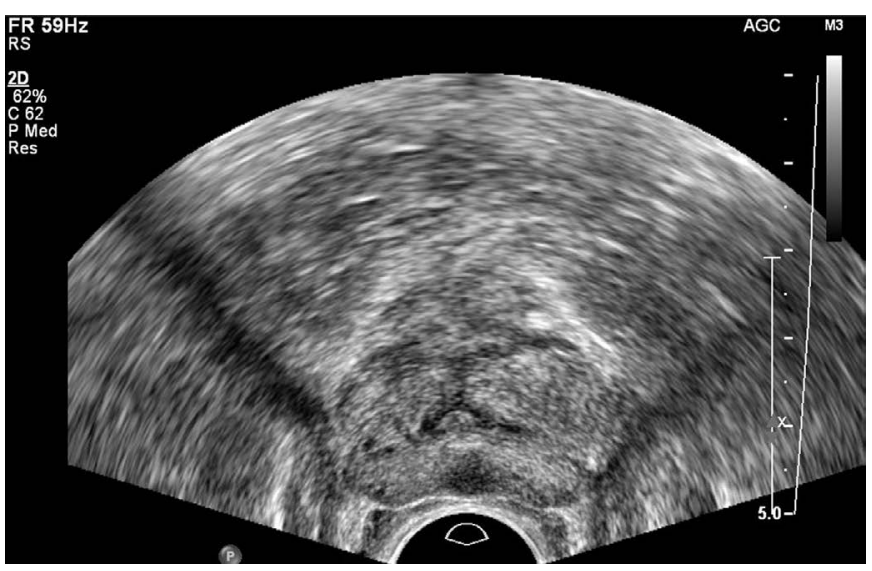

A

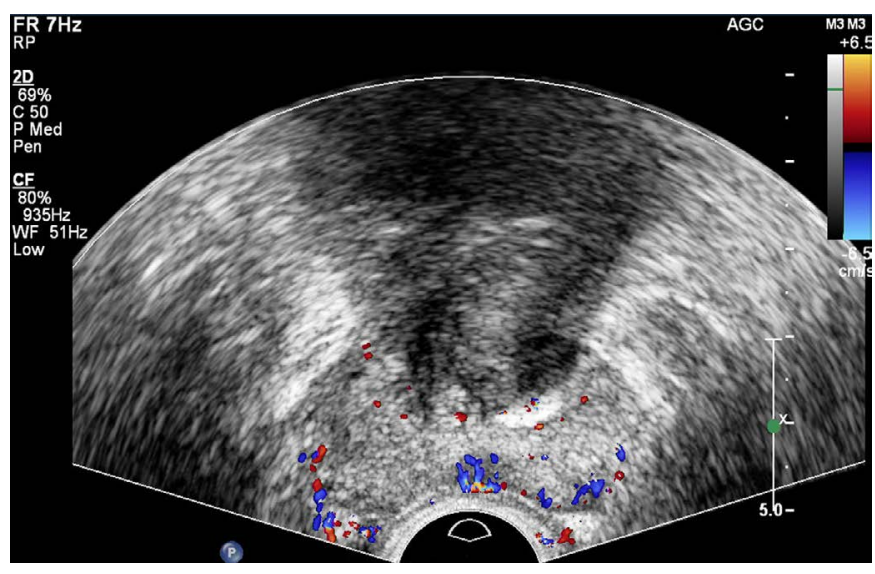

B

Fig. 3. A 62-year-old man with a $0.6 \mathrm{~cm}$ midline focal lesion at mid-gland level on transrectal ultrasonography.

A, B. A nodular lesion with a bulging contour and involved margin is seen. A hypoechoic rim is absent (A). This lesion also shows blood flow in color Doppler ultrasonography (B). Cancer tissue was only positive on targeted biopsy. This patient was diagnosed with clinically significant prostate cancer.

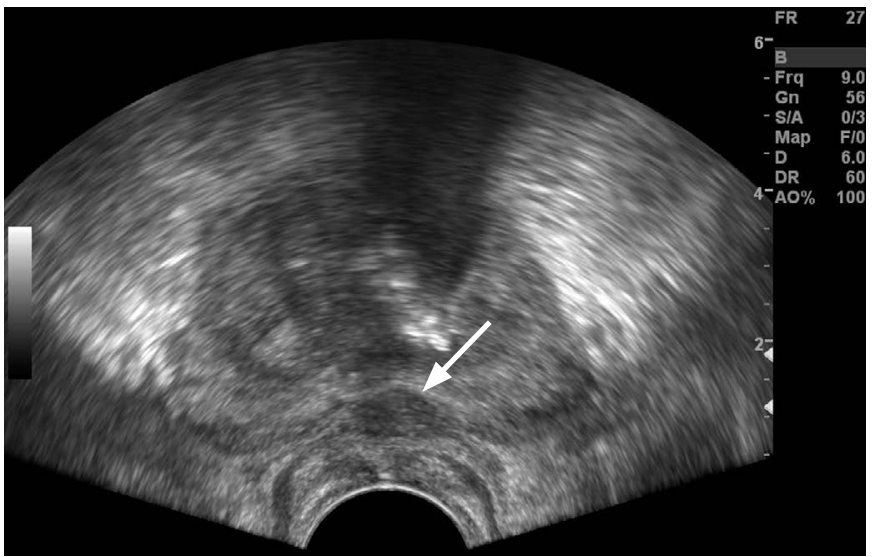

A

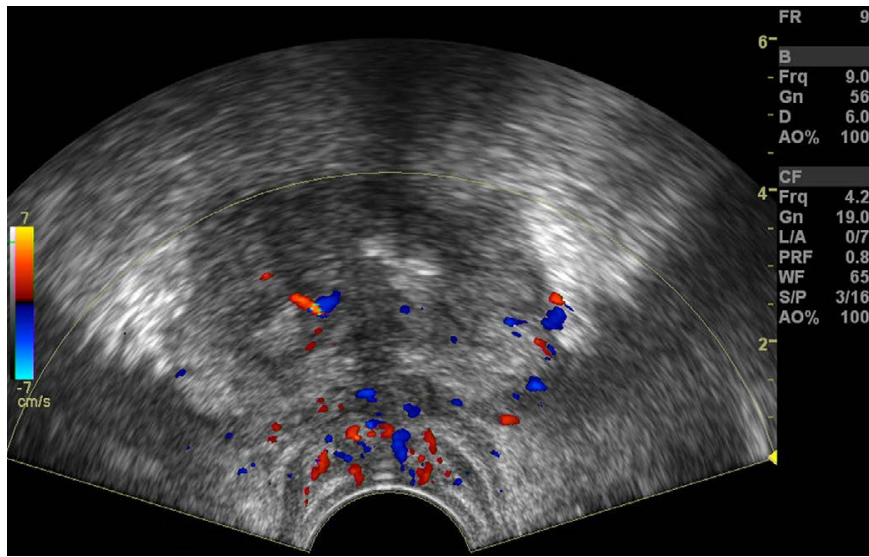

B

Fig. 4. A 74-year-old man with a $1.2 \mathrm{~cm}$ midline focal lesion at the basal level on transrectal ultrasonography.

$A, B$. This is a nodular lesion showing a bulging contour, a clear margin, and the presence of a hypoechoic rim (arrow) (A) and blood flow in color Doppler ultrasonography (B). This patient was diagnosed with clinically insignificant prostate cancer on systematic biopsy, while the targeted biopsy was negative. 
hypoechoic lesions are superior to random systematic biopsies $[15,16]$. Lan et al. [15] concluded that additional targeted biopsies for lesions that are morphologically suspicious for cancer, detected on TRUS, were more likely to improve cancer detection. In our study, focal midline lesions with specific characteristics were proved to be malignant on biopsy results.

Second, the imaging findings of our study support previous study results on the US characteristics of focal prostate lesions. Benign lesions, such as hyperplastic nodules, are frequently depicted as well circumscribed and ovoid-shaped, with a bulging prostate capsule [17]. Hypoechoic perilesional rims around focal lesions can be seen in benign prostatic hyperplasia in the central zone, or in chronic prostatitis in the peripheral zone [18]. Lee et al. [19] reported that irregular margins were observed for malignant lesions, with statistical significance.

According to previous studies and the present findings, midline focal lesions that show nodularity, in the presence of hypoechoic perilesional rims, could be considered benign lesions, such as prostatic inflammation [7] or benign prostatic hyperplastic nodules [17] (Fig. 4). A midline focal lesion with involved margins and the lack of a hypoechoic perilesional rim could be interpreted as a lesion with a relatively high probability of malignancy.

Some limitations of our study should be considered. First, due to the retrospective design, selection bias might have occurred. Second, the small number of subjects could weaken the statistical power. Third, each US criterion was not independent from the other criteria, so statistical confounding could have influenced our results. The use of contrast enhanced US imaging might have improved cancer detection and provided further characterization of midline focal lesions $[10,12]$. Fourth, there is a possibility that core biopsies cannot represent the general characteristics of midline focal lesions. Fifth, pathologic discrepancies between core biopsies and prostatectomy specimens should be considered. Two studies showed upgrading or downgrading of GSs, which could have an effect on clinical significance and therapeutic options for the patients $[20,21]$.

In conclusion, the finding of midline focal lesions of the prostate can contribute to the detection of prostate cancer. The finding of midline focal lesions with involved margins and longer diameters, but without hypoechoic rims, should encourage the clinician to perform targeted biopsies.

ORCID: Junwoo Kim: http://orcid.org/0000-0001-8680-658X; Sung II Hwang: http:// orcid.org/0000-0001-7516-5369; Hak Jong Lee: http://orcid.org/0000-0003-08587873; Sung Kyu Hong: http://orcid.org/0000-0002-8344-6774; Seok-Soo Byun: http://orcid.org/0000-0001-7979-9035; Sangchul Lee: http://orcid.org/0000-00030844-6843; Gheeyoung Choe: http://orcid.org/0000-0001-6547-5603

\section{Conflict of Interest}

No potential conflict of interest relevant to this article was reported.

\section{Acknowledgments}

This research was supported by the Basic Science Research Program through the National Research Foundation of Korea (NRF), funded by the Ministry of Science, ICT and Future Planning (NRF2013R1A1A2011398).

\section{References}

1. Hamper UM, Sheth S, Walsh PC, Holtz PM, Epstein JI. Stage B adenocarcinoma of the prostate: transrectal US and pathologic correlation of nonmalignant hypoechoic peripheral zone lesions. Radiology 1991;180:101-104.

2. Ozden E, Gogus C, Karamursel T, Baltaci S, Kupeli S, Gogus 0 . Transrectal sonographic features of prostatic intraepithelial neoplasia: correlation with pathologic findings. J Clin Ultrasound 2005;33:5-9.

3. Rosenkrantz AB, Taneja SS. Radiologist, be aware: ten pitfalls that confound the interpretation of multiparametric prostate MRI. AJR Am J Roentgenol 2014;202:109-120.

4. Somford DM, Vreuls W, Jansen TS, van Basten JP, Vergunst $H$. Incremental value of transition zone and midline apical biopsy at baseline TRUS-guided biopsy for prostate cancer detection. World J Urol 2014;32:461-467.

5. Ukimura O, Coleman JA, de la Taille A, Emberton M, Epstein JI, Freedland SJ, et al. Contemporary role of systematic prostate biopsies: indications, techniques, and implications for patient care. Eur Urol 2013;63:214-230.

6. Guichard G, Larre S, Gallina A, Lazar A, Faucon H, Chemama S, et al. Extended 21-sample needle biopsy protocol for diagnosis of prostate cancer in 1000 consecutive patients. Eur Urol 2007:52:430-435

7. Rifkin MD, Choi H. Implications of small, peripheral hypoechoic lesions in endorectal US of the prostate. Radiology 1988;166:619622.

8. Linden RA, Halpern EJ. Advances in transrectal ultrasound imaging of the prostate. Semin Ultrasound CT MR 2007;28:249-257.

9. Spajic B, Eupic H, Tomas D, Stimac G, Kruslin B, Kraus O. The incidence of hyperechoic prostate cancer in transrectal ultrasoundguided biopsy specimens. Urology 2007;70:734-737.

10. Heijmink SW, van Moerkerk H, Kiemeney LA, Witjes JA, Frauscher $F$, Barentsz JO. A comparison of the diagnostic performance of systematic versus ultrasound-guided biopsies of prostate cancer. Eur Radiol 2006;16:927-938.

11. Halpern EJ, Strup SE. Using gray-scale and color and power Doppler sonography to detect prostatic cancer. AJR Am J Roentgenol 2000;174:623-627. 
12. Raja J, Ramachandran N, Munneke G, Patel U. Current status of transrectal ultrasound-guided prostate biopsy in the diagnosis of prostate cancer. Clin Radiol 2006;61:142-153.

13. Scattoni V, Zlotta A, Montironi R, Schulman C, Rigatti P, Montorsi F. Extended and saturation prostatic biopsy in the diagnosis and characterisation of prostate cancer: a critical analysis of the literature. Eur Urol 2007;52:1309-1322.

14. Lee HJ, Kim KG, Lee SE, Byun SS, Hwang SI, Jung SI, et al. Role of transrectal ultrasonography in the prediction of prostate cancer: artificial neural network analysis. J Ultrasound Med 2006;25:815821.

15. Lan SK, Tsai YS, Lin YH, Tzai TS. Diagnostic performance of a random versus lesion-directed biopsy of the prostate from transrectal ultrasound: results of a 5-year consecutive clinical study in 1 institution in South Taiwan. J Ultrasound Med 2007;26:11-17.

16. Toi A, Neill MG, Lockwood GA, Sweet JM, Tammsalu LA, Fleshner NE. The continuing importance of transrectal ultrasound identification of prostatic lesions. J Urol 2007;177:516-520.

17. Oyen RH, Van de Voorde WM, Van Poppel HP, Brys PP, Ameye $F E$, Franssens $Y M$, et al. Benign hyperplastic nodules that originate in the peripheral zone of the prostate gland. Radiology 1993; 189:707-711.

18. Lee HJ, Choe GY, Seong CG, Kim SH. Hypoechoic rim of chronically inflamed prostate, as seen at TRUS: histopathologic findings. Korean J Radiol 2001;2:159-163.

19. Lee HY, Lee HJ, Byun SS, Lee SE, Hong SK, Kim SH. Classification of focal prostatic lesions on transrectal ultrasound (TRUS) and the accuracy of TRUS to diagnose prostate cancer. Korean J Radiol 2009;10:244-251.

20. Cohen MS, Hanley RS, Kurteva T, Ruthazer R, Silverman ML, Sorcini $A$, et al. Comparing the Gleason prostate biopsy and Gleason prostatectomy grading system: the Lahey Clinic Medical Center experience and an international meta-analysis. Eur Urol 2008;54:371-381.

21. Kuroiwa K, Shiraishi T, Naito S; Clinicopathological Research Group for Localized Prostate Cancer Investigators. Gleason score correlation between biopsy and prostatectomy specimens and prediction of high-grade Gleason patterns: significance of central pathologic review. Urology 2011;77:407-411. 